\title{
Automatic Generation of Chaotic Attractors with Various Cyclic or Dihe- dral Symmetries
}

\author{
Zhihui Wang ${ }^{\mathrm{a}}$, Shitao Yan $^{\mathrm{b}}$ and Peichang Ouyang, ${ }^{\mathrm{c},}$ \\ ${ }^{a}$ Handan College, Handan, China; ${ }^{b}$ Henan Institute of Science and Technology, Xinxiang, China; ${ }^{c}$ School of Mathemat- \\ ics and Physics, Jinggangshan University
}

\begin{abstract}
Chaotic attractors are created by iterating functions that are equivariant with respect to the cyclic or dihedral groups. An improved color scheme based on the visit frequency of the pixels is proposed to render chaotic attractors. By normalization and scale transformation, aesthetic patterns which simultaneously have several kinds of cyclic or dihedral symmetries are generated. This method can be used to yield a great number of aesthetic patterns automatically.
\end{abstract}

Keywords: Chaotic attractor, symmetry, cyclic group, dihedral group.

\section{INTRODUCTION}

Kinds of the symmetry groups in the Euclidean plane are well understood [1]. Up to isomorphism, they are classified as cyclic or dihedral groups, frieze groups and wallpaper groups. It is surprising that chaotic attractor is compatible with symmetry. Automatic generation of symmetrical patterns has been an active topic of recent study [2-7]. There are many methods dedicated to yield patterns with cyclic or dihedral symmetry. For example, Field and Golubitsky constructed polynomials that are equivariant with respect to these groups and created a great many of chaotic attractors [2]. Reiter proposed a convenient group summation technique to generate similar planar or three-dimensional attractors [8]. Colored cyclic or dihedral patterns were produced by Lu et al. from a dynamic systems' point of view.

In [9], a smart way was established to yield interesting dueling chaotic attractors. In that paper, two functions of the plane having distinct symmetries are intertwined using a sinew function to create a function of three variables having different symmetries in different regions. However, this strategy is no longer proper for lager $k$ ( $k$ is the number of cyclic or dihedral symmetries of the attractor). The reason is that, on the one hand, the construction of sinew function would become difficult since it should simultaneously satisfy several different kinds of symmetries. On the other hand, even if one could construct similar sinew functions, the color scheme employed in [9] is no longer proper to produce appealing patters since the constructed function would be quite complex.

This paper article concerns with generating beautiful patterns which simultaneously have several kinds of cyclic or dihedral symmetries. The remainder of this paper is organized as follows. In Section 2, we introduce how to create chaotic attractors with desired symmetries. In Section 3, we present the method of generating chaotic attractors that have several kinds of cyclic or dihedral symmetries. In Section 4, we show some aesthetic patterns and detailed implements.

\section{FUNCTIONS THAT ARE EQUIVARIANT WITH RESPECT TO CYCLIC OR DIHEDRAL GROUPS}

The cyclic group is generated by $n$-fold rotations about a single point. The dihedral group contains those rotations and reflections through the point of rotation. The completeness enumeration of groups andis called Leonardo's Theorem [10].

In order to generate attractors with specified symmetries, we need functions that preserve symmetries in a certain manner. In particular, A function is said to be equivariant with respect to the symmetries of $\mathrm{G}$ if for all, where.

Letbe a rotation and be anequivariant function with respect to. Then the iteration of the rotation of a point is the same as the rotation of the iteration of the point. This means that the attractors associated with tends to have the desired symmetries.

In [2], Field and Golubitsky constructed a truncated equivariant complex function of the form

where is the real part of complex, and are complex constants. (1) is equivariant with respect to dihedral symmetry when.

Proposition 1. Let be an arbitrary function, be a finite group realized by matrices acting on. Assume is a function defined as

Then, is equivariant with respect to.

The proof of Proposition 1 can be found in [8]. It was proposed by Reiter to create chaotic attractors with cyclic or dihedral symmetry. In this paper, we adopt formula (2) instead of (1) since the freedom ofin (2) would provide great convenience for creating chaotic attractors. We next give the matrix presentations ofand. 
Let and be, respectively, cyclic and dihedral groups. Denote and. Then is the generator of and is the realization of. We will consider being equal to that set of matrices. Since is a reflection through the origin, set is a presentation of group.

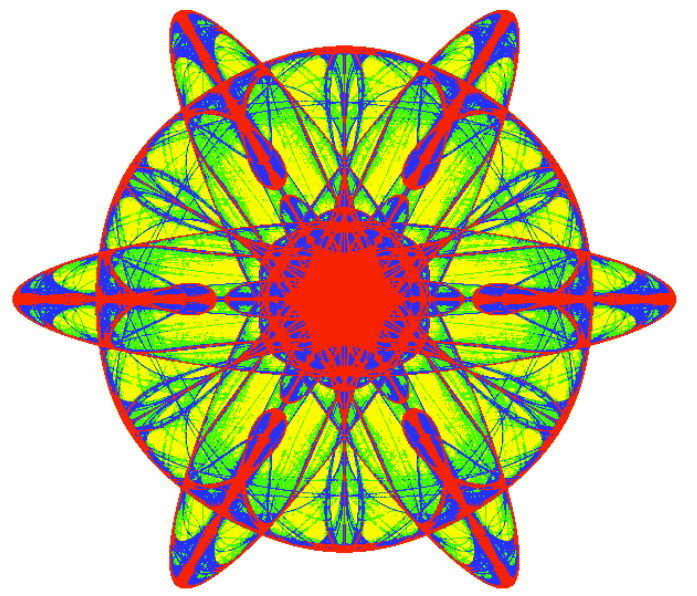

Fig. (1). A chaotic attractor with symmetry.

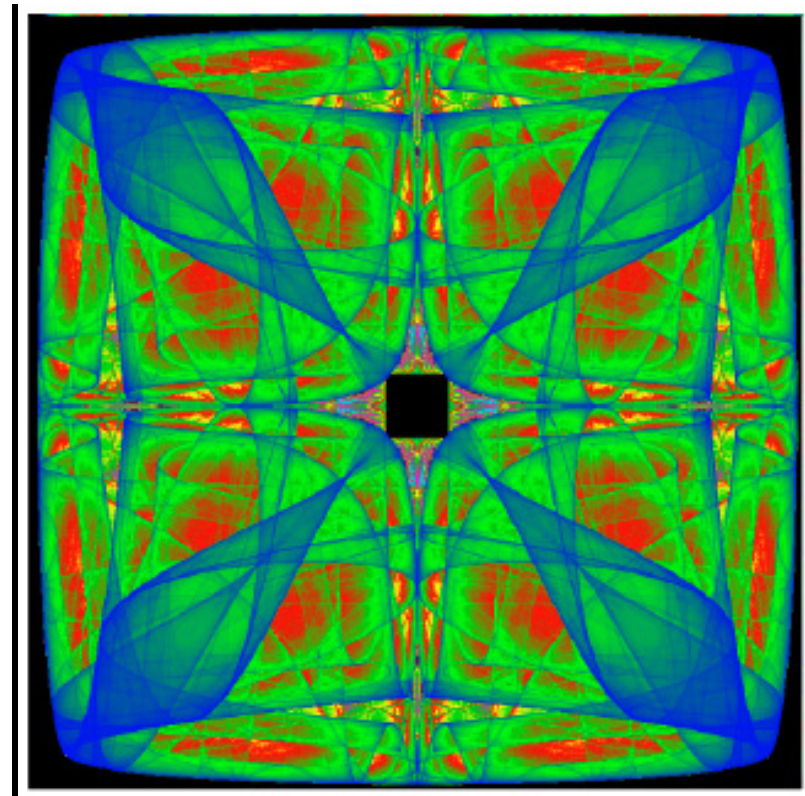

Fig. (2). A chaotic attractor with symmetry.

In Proposition 1, letor. Then, the attractor generated bywill possess cyclic or dihedral symmetry. Figs. (1) and (2), respectively, show two chaotic attractors generated byand .

\section{ALGORITHM FOR GENERATING ATTRACTORS WITH SEVERAL CYCLIC OR DIHEDRAL SYMME- TRIES}

It is well known that global cyclic or dihedral symmetries are incompatible in a pattern except for trail situation. Here "the pattern with several kinds of cyclic or dihedral symmetries" means that different ring regions have different cyclic or dihedral symmetries. We use basic geometric transformations to achieve the target.

Fig. (3) shows how to transform an attractor into specified ring region. At the top of Fig. (3), assume the yellow pentagon is a chaotic attractor centered at origin with range.
Let and be the radii of circles. First, is normalized into circles of radius, see the middle of Fig. (3). Then, by translation and scale transform, the normalized attractor is transformed into the target region, see the bottom of Fig. (3). We summarize the detailed method as follows:

Step 1. By proposition 1, create an attractor with cyclic or dihedral cyclic or dihedral symmetry.

Step 2. Assume is a point of attractor, let. For, denote. Then is a point of unit disc and we get the normalized attractor.

Step3. Assume is scaling factor. For, denote.

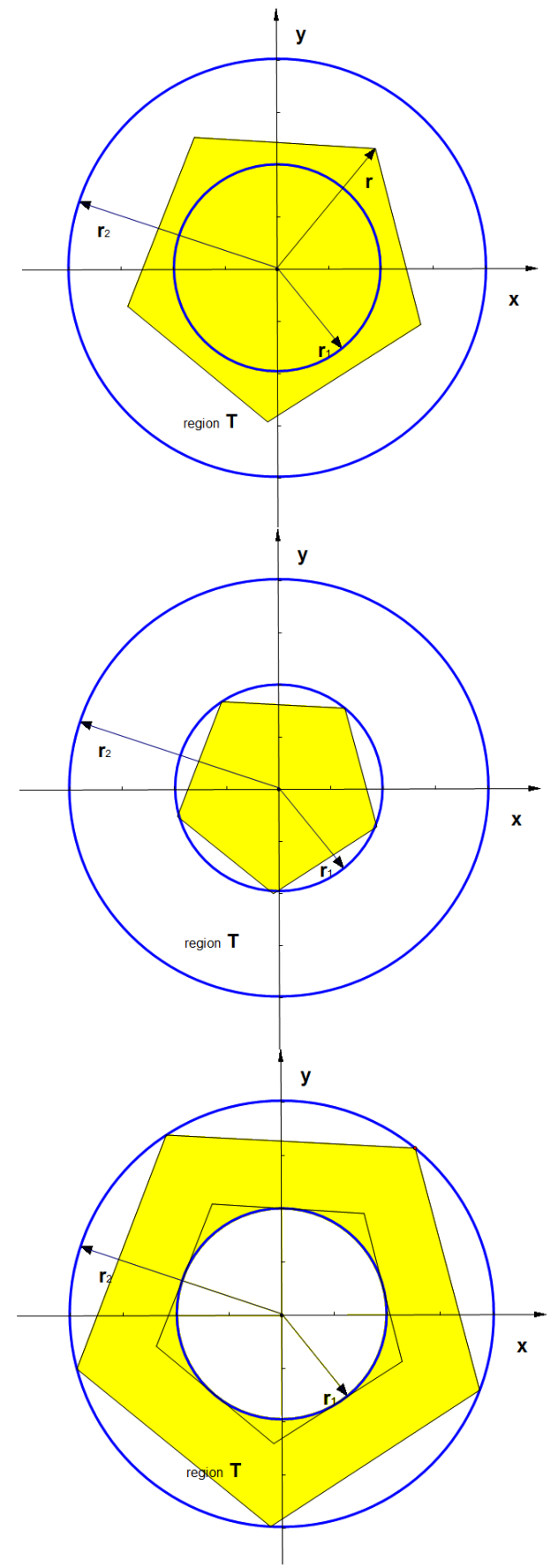

Fig. (3). Schematic diagram that transforms an attractor into the desired ring region. 
Step 4. Let be a new attractor, be a ring region adjacent to. With a similar process used in Step 1-3, we can transform to. If we repeat this algorithm times, we thus obtain a pattern with kinds of cyclic or dihedral symmetries.

In Fig. (4), we show a ring pattern of symmetry obtained by this method.

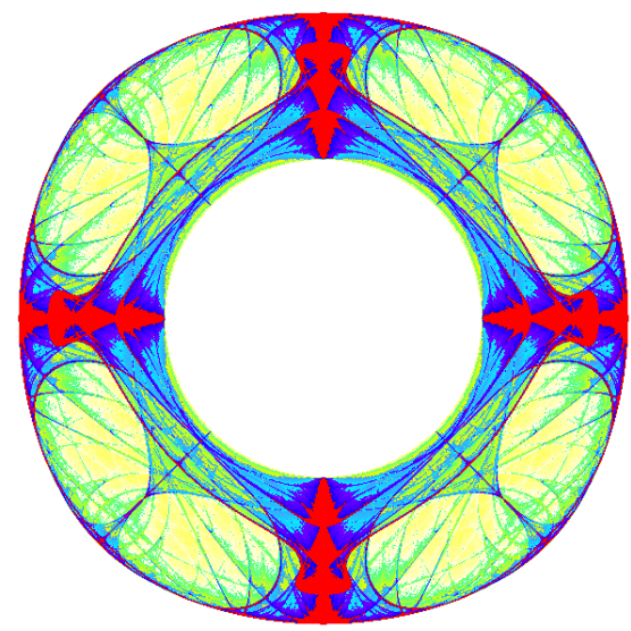

Fig. (4). An annular attractor with symmetries transformed by Steps $1-3$.

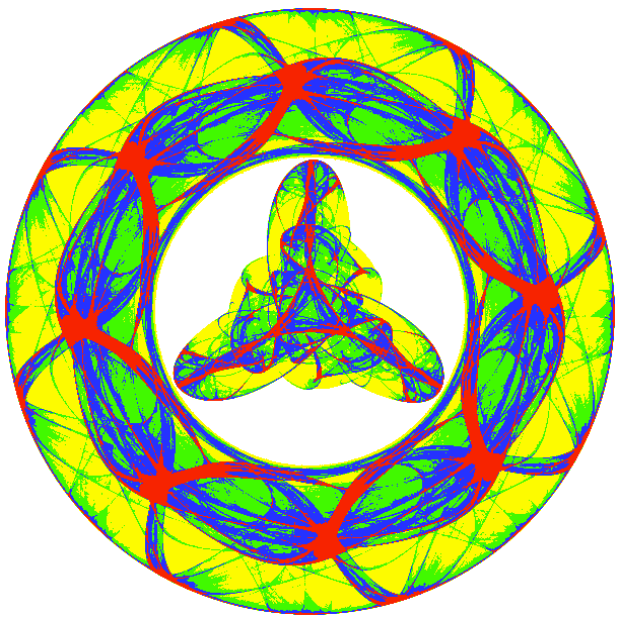

Fig.(5). An aesthetic pattern with symmetry.

\section{EXAMPLES AND IMPLEMENTS}

In this section, we show some aesthetic patterns and implements used in generating attractors.

The color scheme based on visit frequencies of the pixels is the most popular method in rendering chaotic attractor. We also employ this scheme to color attractors. For more details of the scheme, please refer to [2-5, 11-13].

The accurate $r$ used in Step 2 is difficult to find since our systems are all chaotic. In practice, we iterated equivariant functions for 1000 times and selected the maximum module asof Step 2. Our experiment results showed that this method served well.

All the functions used in this paper are trigonometric functions. For example, the function used to create Fig. (1) is.

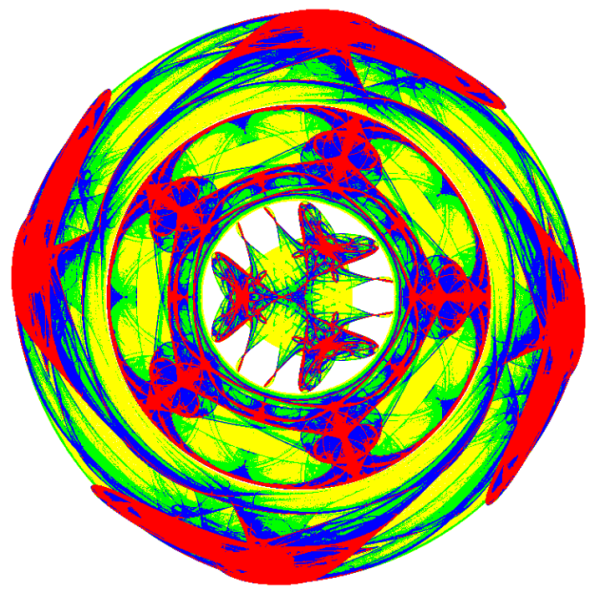

Fig. (6). An aesthetic pattern with symmetry.

To yield visually appealing attractors, we computed the Ljapunov exponent [14] in advance and selected functions that have positive Ljapunov exponent.

Attractors shown in this paper were obtained by iterating equivariant functions over 200 million times. We have arranged that the ring width $S$ in Step 3 is always 1. For convenience, the symbol represents that, from inner to outer, the corresponding pattern hassymmetries respectively. In Figs. (4-7), we show four resulting patterns of this kind.

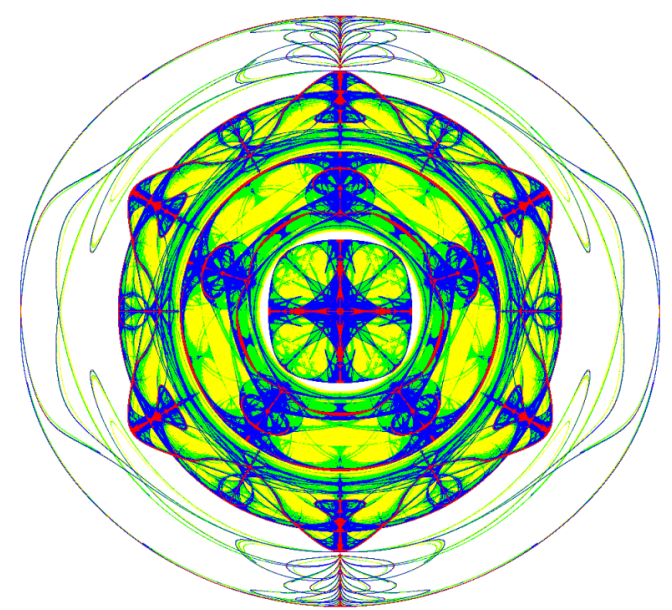

Fig. (7). An aesthetic pattern with symmetry.

\section{CONCLUSITON}

Automatic generation of chaotic attractors with several kinds of cyclic or dihedral symmetries method is realized by normalization, translation and scale transform techniques. The traditional color scheme used to render chaotic attractor is based on the frequency of the pixels visited. We improve it and develop a more efficient and convenient approach to color chaotic attractors. The algorithm can accurately control the color percentage of an image, which shows great convenience in rendering chaotic attractors. Our method can be applied to create a great many of beautiful patterns automatically.

\section{CONFLICT OF INTEREST}

The authors confirm that this article content has no conflict of interest. 


\section{ACKNOWLEDGEMENTS}

This work was supported by the Natural Science Foundation of China (No. 11461035), Natural Science Foundation of Jiangxi province of China (No. 20142BAB211012).

\section{REFERENCE}

[1] H.S.M. Coxeter, W.O.J. Moser, Generators and Relations for Discrete Groups. Springer-Verlag, New York, 1980.

[2] M. Field, M. Golubitsky, Symmetry in Chaos. Oxford University Press, 1992.

[3] N. Carter, R. Eagles, S. Grimes, A. Hahn, C. Reiter, "Chaotic attractors with discrete planar symmetries," CHAOS SOLITON FRACT, vol. 12, pp. 2031-2054, 1998.

[4] N. Carter, C. Reiter, "Frieze and wallpaper chaotic attractors with a polar spin," COMPUT GRAPH-UK, vol. 22, pp.765-779.

[5] J. Dumont, F. Heiss, K. Jones, C. Reiter, L. Vislocky, "Chaotic attractors and evolving planar symmetry," COMPUT GRAPH-UK, vol. 23, pp. 613-619, 1999.
[6] K.W. Chung, H.S.Y. Chan, "Symmetrical patterns from dynamics," COMPUT GRAPH FORUM, vol. 12, pp. 33-34, 1993.

[7] J. Lu, Z.X. Ye, Y.R. Zou, R.S. Ye, "Orbit trap rendering methods for generating artistic images with crystallographic symmetries," COMPUT GRAPH-UK, vol. 29, pp.787-794.

[8] K. Jones, C. Reiter, "Chaotic attractors with cyclic symmetry revisited," COMPUT GRAPH-UK, vol. 24, pp. 271-282, 2000.

[9] C. Reiter, "Attractors with dueling symmetry," COMPUT GRAPHUK, vol. 21, pp. 263-271, 1997.

[10] E. George, The Foundations of Geometry and the Non-Euclidean Plane. Springer-Verlag, New York, 1986.

[11] N. Chen, D. Hao, M. Tang, "Automatic generation of symmetric IFS contracted in the hyperbolic plane," CHAOS SOLITON FRACT, vol. 41, pp. 829-842, 2009.

[12] J. Dumont, F. Heiss, K. Jones, C. Reiter, L. Vislocky, "Ndimensional chaotic attractors with crystallographic symmetry," CHAOS SOLITON FRACT, vol. 12, pp. 2761-784, 2001

[13] C. Reiter, "Chaotic attractors with the symmetry of tetrahedron," COMPUT GRAPH-UK, vol. 21, pp. 841-848, 1997.

[14] T. Parker, L. Chua, Practical numerical algorithms for chaotic systems. Springer -Verlag, New York, 1989.

(C) Wang et al.; Licensee Bentham Open.

This is an open access article licensed under the terms of the Creative Commons Attribution Non-Commercial License (http://creativecommons.org/licenses/by-nc/3.0/) which permits unrestricted, non-commercial use, distribution and reproduction in any medium, provided the work is properly cited. 